\title{
The application of a free-swimming ROV in aquaculture
}

\author{
R. A. KLEPAKER, K. VESTGÅRD $\dagger$ \\ J. O. HALLSET and J. G. BALCHEN
}

Keywords: Freeswimming ROV, acoustic command telemetry link, acoustic navigation

In 1985, SINTEF and SIMRAD Subsea A/S started to develop an autonomous free swimming vehicle. The project was to develop a prototype of a small vehicle, in order to obtain knowledge and experience in designing, controlling and operating such vehicles. This was ready for testing at the end of 1985 . The vehicle is controlled by an acoustic data telemetry system. The vehicle has a built-in television camera and containers for other sensors. It is suitable for inspection purposes. This paper describes the vehicle and some of the principles used.

\section{Introduction}

SIMRAD Subsea $\mathrm{A} / \mathrm{S}$ has for many years designed and manufactured systems for underwater operations based on underwater acoustics. The knowledge and experience SIMRAD Subsea $\mathrm{A} / \mathrm{S}$ has in acoustic signal processing, and knowhow and ideas from SINTEF was a stimulant to start the development of a freeswimming, tetherless underwater vehicle. The project has been going on at SINTEF and SIMRAD Subsea A/S since 1984. We now have a prototype that forms a basis for a freeswimming underwater vehicle concept. This prototype system will be subject to extensive testing of applied principles.

In order to achieve a commercial system further developments are going on. A major part of the project is a fast acoustic sensor-data telemetry link. This is necessary in order to get sensor-data, such as TV-pictures, to the surface. The other project will be the adaptation of the fisheries research equipment that SIMRAD Subsea $\mathrm{A} / \mathrm{S}$ manufactures.

The system will be well suited for inspection purposes in fields as offshore, naval and oceanographic fisheries research. We believe that the aquaculture market will also need such a freeswimming vehicle system. Therefore, we describe the vehicle and some sensors that may be of interest in aquaculture in the future.

\section{System}

The prototype system consists of a tetherless underwater vehicle, an acoustic commandlink, an underwater navigation system and an operator's unit. The commercial version will include a number of sensors and a sensor data acoustic telemetry link. The vehicle is autonomous and will receive its commands from the operator via the operator's unit and the command telemetry link.

Received 15 January 1987.

This paper was presented at the IFAC Symposium on Automation and Dataprocessing in Aquaculture, Trondheim, Norway, 18-20 August 1986.

This paper is reprinted with the permission of IFAC.

† SIMRAD Subsea A/S, 3191 Horten, Norway.

‡ SINTEF, avd. 48, 7034 Trondheim NTH, Norway. 


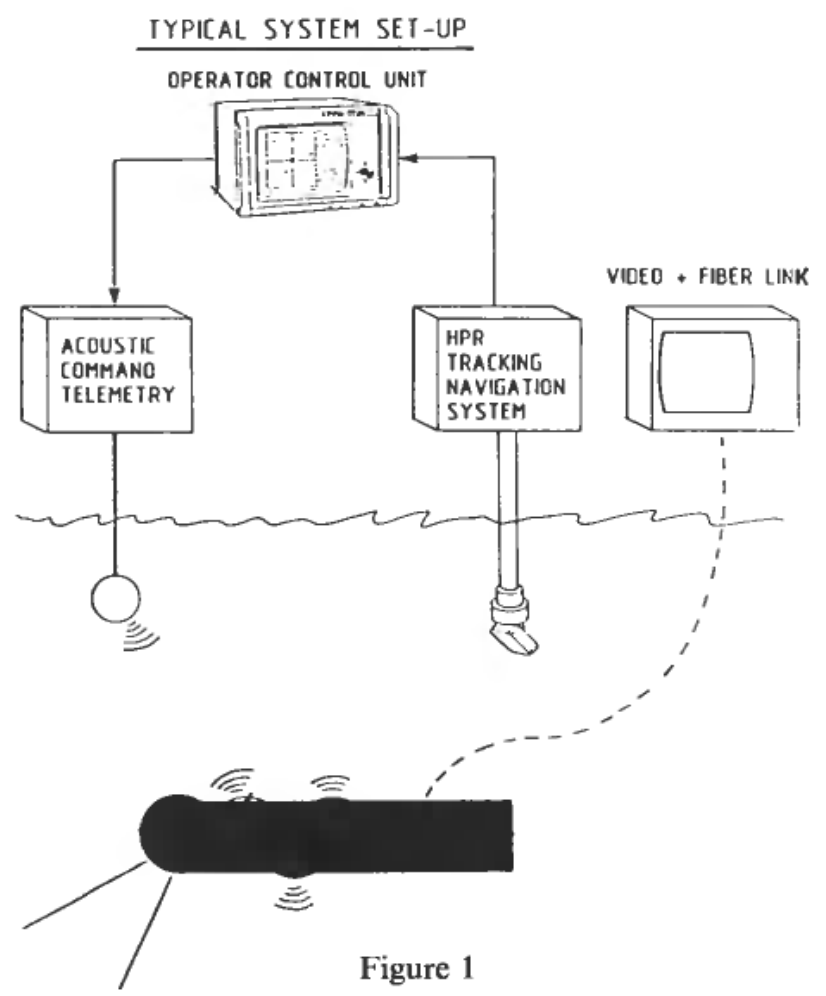

The present vehicle can perform the following functions:

(a) swim or stay at a desired depth of water

(b) swim or stay at a desired distance above the bottom

(c) swim at a certain speed in its $\mathrm{X}$ or $\mathrm{Y}$ direction desired

(d) swim on a desired course

(e) operate the different sensors that may be onboard.

Figure 1 shows a typical ROV operation where a surface vessel supports the ROV. The surface vessel has a 'Super Short Baseline' tracking navigation system that gives the position of the underwater vehicle with reference to the surface vessel or/and to any structure in the water marked with a transponder. Also the underwater vehicle has a transponder as a navigation reference. The operator's unit is integrated with the navigation system, and his display shows the underwater situation and a vehicle control/command menu. Figure 2 shows a situation where the vehicle is deployed from a pier and the navigation transducer(s) is mounted on the pier or at positions that makes it possible to cover the complete area in which the vehicle will operate.

\subsection{System specifications \\ ROV (vehicle) \\ Max depth $\quad 500 \mathrm{~m}$ \\ Max speed 4.5 knots \\ Endurance at $1.5 \mathrm{knot} \quad 5$ hours \\ Weight $250 \mathrm{~kg}$}




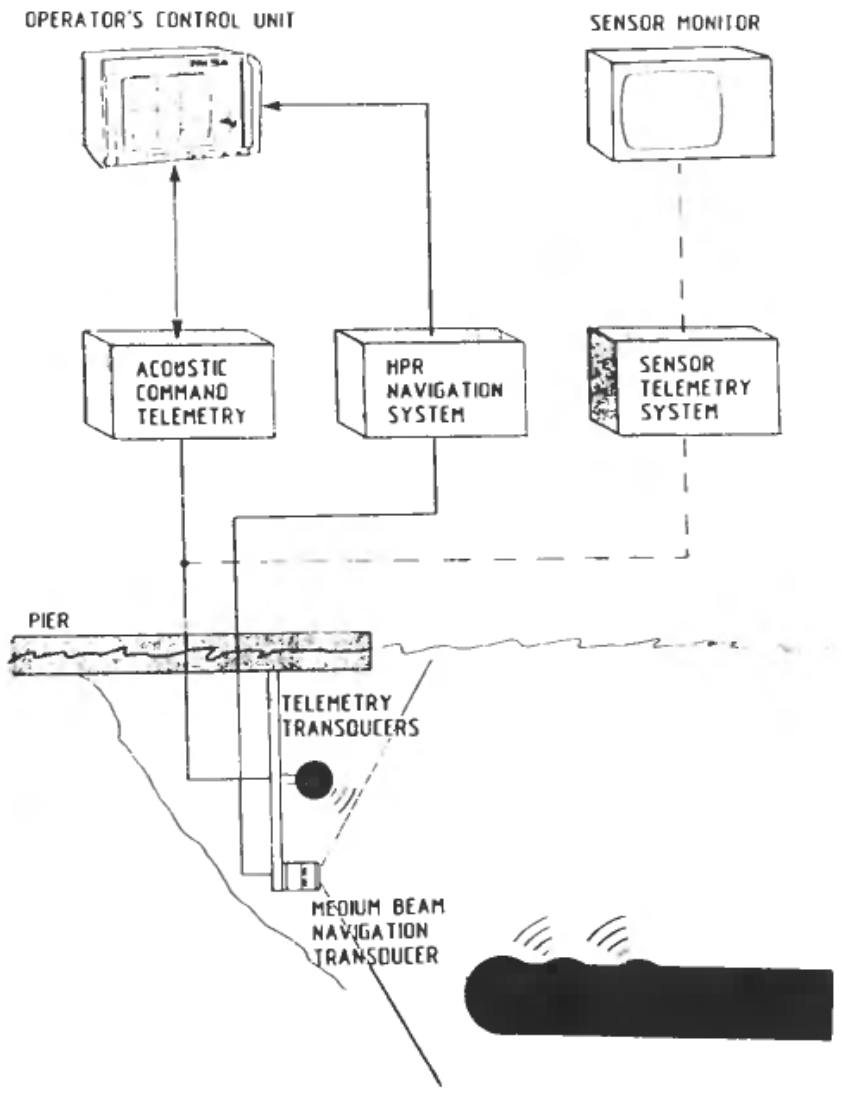

Figure 2

Acoustic command link

$\begin{array}{ll}\text { Max range } & 700 \mathrm{~m} \\ \text { Bit rate } & 50 \mathrm{bit} / \mathrm{sec} . \\ \text { Frequency range } & 65-75 \mathrm{kHz} \\ \text { Navigation system } & \\ \text { Max range } & 1000-1500 \mathrm{~m} \\ \text { Accuracy } & 1 \% \text { of range } \\ \text { Frequency range } & 20-30 \mathrm{kHz}\end{array}$

\section{Vehicle}

The vehicle is streamlined in order not to be trapped in any ropes, nets, wires or structures in the water. All components except the main propellers are located inside the fibreglass hull. The vehicle is moved ahead or astern by two main propellers and sideways by a side thruster. The main propellers are located in each rear corner of the vehicle, and the side thruster is in the middle of the vehicle. The vertical force is obtained by means of a swimbladder. In addition, a height rudder can be used for climbing/decoding. In order to reduce weight and volume, all motors for propulsion as well as servos are pressure compensated by the use of $\mathrm{N}_{2}$ gas. The gas supplies are stored in two gas cylinders. Figure 3 shows a block diagram of the vehicle. 


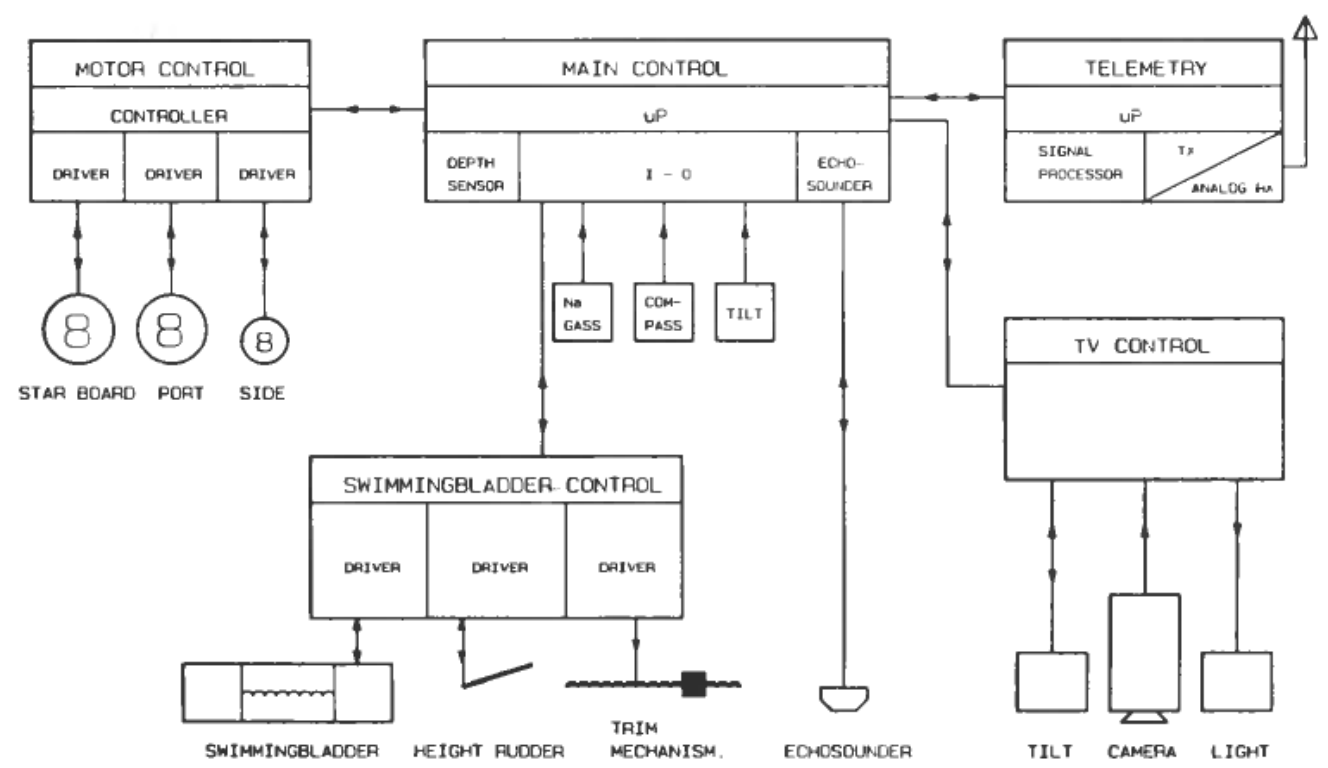

Figure 3. Block diagrams of the ROV.

\subsection{Control system}

The main control system is housed in an aluminium container. The system consists of a microprocessor and some interface circuits. By means of input data from the command system and sensors and calculations carried out by the microprocessor, the control system determines the required volume of the swimbladder, the necessary power to the different propellers, the depth rudder angle and the trim mechanism data.

The control system has algorithms for depth control, speed and course. A mathematical model of the vehicle is included into the control system.

Information from different sensors such as echo sounder, depth sensor, gas sensor, compass and tilt sensors are fed into the control system. Commands and parameters, from the operator, comes to the control system via the acoustic command telemetry system.

The operator can ask for status readback from the vehicle. The data is transferred to the surface via the acoustic command telemetry link.

An important function is an automatic safety system. If the transmission between the operator and the vehicle fails for a certain time, the vehicle stops and tries to keep station. If nothing happens for a longer time, the vehicle will return to the surface. If the gas-supply or the battery power is reaching a lower threshold, the vehicle will alert the operator and then return to the surface.

\subsection{Motor system}

Inside the vehicle are 7 motors. There are 3 for propulsion, and 4 servo motors for the swimbladder, the height rudder, the trim mechanism and the TV control mechanism. All motors are housed in thin containers that are pressure compensated. They are filled with nitrogen from a gas system. All motors are speed controlled.

The propulsion motors are geared to the propellers which are located in each rear corner of the vehicle. A side thruster is located in a tunnel through the centre of the vehicle. 
Course control is made by giving the two main propellers different speeds. Sideways movement is controlled by the side thruster. The three propellers are controlled from the motor control container. This communicates with the main control container.

\subsection{Battery system}

The necessary DC-power for propulsion and instrumentation is supplied by three lead accumulators. They are sealed and pressure compensated by filling them with oil. Two accumulators produce $24 \mathrm{~V}$ for propulsion and the servos. The third battery gives $12 \mathrm{~V}$ to the other electronic circuits, viz. the control system, the telemetry system, the TV system and optimal sensors.

\subsection{Swimming bladder and gas system}

A 'swimbladder' is installed to effect vertical movement. The volume of the swimbladder is controlled by a servo system. The swimbladder is filled with $\mathrm{N}_{2}$ the pressure of which is constant and slightly exceeds the ambient pressure. To decrease the volume, $\mathrm{N}_{2}$ must be drained out, to increase the volume $\mathrm{N}_{2}$ must be supplied. 201 of $\mathrm{N}_{2}$ at 200 bar is stored in two gas-cylinders. The gas system is connected to the swimbladder as well as to all the motors.

The gas pressure into these units is adjusted by a special valve in order to keep the pressure constantly relative to the ambient hydrostatic pressure. The gas pressure is watched by the control system. The gas system is completely independent of the electronics so that the pressurized components will not be damaged if some electronics should fail or the batteries are discharged.

\section{Sensors}

The ROV can be equipped with a number of sensors or instruments for different applications. For aquaculture the sensors will be a TV-camera, an echo integrator and split beam echo sounder. There are two containers in the vehicle that can house the necessary electronics, and the hull has spare space for several transducers. The advantage of carrying the sensors on such a vehicle is that it does not affect the fish's behaviour. In this manner more accurate measurements can be made. Sensor data are stored in the vehicle or transferred to the surface via the acoustic sensor data telemetry link. The data may be correlated to the navigation data.

\subsection{TV camera}

This can be tilted $\pm 95^{\circ}$. There are a large number of cameras with different characteristics and specifications. In order not to attract or influence the fish, a minimum of light should be emitted from the vehicle. A low level light camera is mounted on the vehicle. An advantage is the low power such a system drains from the limited power source. Such a camera also gives better pictures than ordinary underwater cameras due to the minimum of light reflections from particles flowing in the water.

\subsection{Echo integrating system}

The method of integrating fish echoes to get a figure for the amount of fish in an area have been used since 1967. This is a reliable method for fish stock assessment 
and should be of interest in larger areas where the fish do not overlap each other. Such a system can be used from surface vessel, but a disadvantage is that the beam from the echo sounder covers just a small area close to the surface. By having the system on the underwater vehicle, the vehicle can follow the bottom and measure the fish amount in the upper part of the sea-volume. Fish in this upper layer are often frightened away from the beam by the noise of the vessel.

\subsection{Split beam echosounder}

SIMRAD Subsea $\mathrm{A} / \mathrm{S}$ has developed equipment for very precise measurements of the size of fish. This is based upon the split beam principle. The system uses a quadruple transducer and separate transceivers on each quadrant, it is able to determine where in the tranducer beam the fish are located. When the angles are known, the echo amplitude can be connected accordingly, in order to have the correct strength of the echo. In this way correct target strength of all fish that pass through the echo sounder beam can be measured.

\section{The acoustic command telemetry link}

The acoustic telemetry system transfers commands from the operator's unit to the underwater vehicle. The operator also needs to have vehicle status readback. The telemetry system is therefore a two-way system that consists of two electronically identical units. Each unit consists of an acoustic signal transceiver, a digital signal processor, a control processor and an omnidirectional transducer. The acoustic coding principle is based upon multifrequency pulse coding in order not to be influenced by interference caused by reflections or multipath sound.

A command/status telegram consists of a series of 12 bit messages. Each message is coded as a sequence of 7 pulses with individual frequencies. A series of messages is separated in frequency using a hopping technique. Three separate sub-bands are available, each with 7 frequency channels. The maximum capacity of the coding principle is $100 \mathrm{bit} / \mathrm{s}$. The ROV link operates with $80 \mathrm{bit} / \mathrm{s}$ for a single message and about $50 \mathrm{bit} / \mathrm{s}$ for a total telegram. Operating frequency bandwidth requirement is $56 \mathrm{~Hz}$, the center frequency is $70 \mathrm{kHz}$.

\section{Navigation}

The underwater vehicle is navigated by the use of a supershort baseline system, a water pressure sensor and an echo sounder. The SSBL system consists of a display unit, an acoustic transceiver, an angle sensor transducer and a transponder, whose position is to be measured.

The angle sensor transducer transmits a pulse to the transponder which in turn replies. That reply is received by the transducer and the acoustic transceiver measures the time between the transmission and the reception of the reply. That time gives the range. The transducer also measures the incident angles (vertical or horizontal) of the incoming sound pulse. Then the position can be calculated.

Due to variations in sound velocity in different layers of water, the path of the reply is bent and an error in the vertical angle reading then occurs. The navigation system can then use the information from the depth sensor or the echo sounder on board the vehicle and correct the measured position to the correct depth. There are 


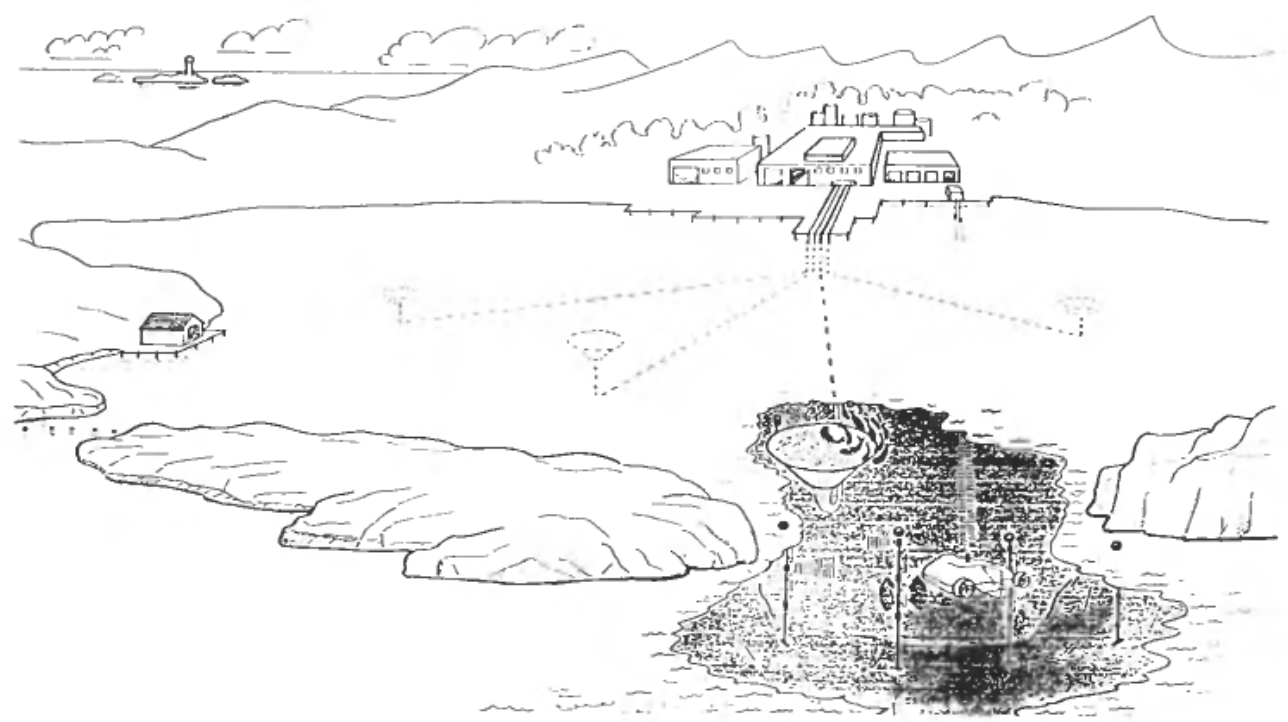

Figure 4

two SSBL systems that work well in this ROV system; a pointing narrow beam system and a fixed medium beam system.

The narrow beam pointing system gives an accuracy better than $1^{\circ}$ of the range in the complete underwater area within a range of $2000 \mathrm{~m}$. This system has a mechanical trained narrow beam transducer with electronic beam steering in the vertical plane. This system is mounted on a surface vessel.

The medium beam system can be mounted on any subsea structure and operates within a cone of \pm 55 degrees. One or more of these underwater transducers can be mounted subsea on a vessel, or at a pier in order to cover the desired area (Fig. 2). This system gives an accuracy of $2 \%$ of the range to the transponder.

\section{Conclusion}

Some future fish farming will be located in fjords, maybe with several inlets. Each inlet will have electrical or acoustical barriers that prevents the fish inside from escaping (Fig. 4). In such a farm several tasks can be carried out by the free swimming vehicle:

Checking the function of the barriers by the use of sonar or TV.

Measuring the fish size and the total amount of fish thus getting a figure of the accession. This is possible by using the split beam echo sounder and the echo integrating system.

Watching the fish behaviour at various places.

General inspection of the plant (pollution, water salinity, temperature, content of oxygen etc.).

The advantage of the ROV is that it provides an instrument platform for in situ observations of fish without affecting their behaviour. 